\title{
SAFELY OPERATED TWO PATIENTS IN PREGNANCY WITH PRIMARY HYPERPARATHYROIDISM
}

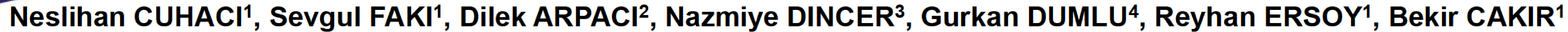

Yildirim Beyazit University, Faculty of Medicine, Ataturk Education and Research Hospital, Department of Endocrinology and Metabolism, Ankara, TURKEY 2 Bulent Ecevit University, Faculty of Medicine, Department of Endocrinology and Metabolism, Zonguldak, TURKEY

${ }^{3}$ Ataturk Education and Research Hospital, Department of Pathology, Ankara, TURKEY

${ }^{4}$ Yildirim Beyazit University, Faculty of Medicine, Ataturk Education and Research Hospital, Department of General Surgery, Ankara, TURKEY

\section{INTRODUCTION}

> Primary hyperparathyroidism (pHPT) during pregnancy is rare and associated with increased morbidity for both mother and fetus.

> Maternal complications of pHPT include nepfhrolithiasis, pancreatitis, cardiac arrhytmias, hypertension, nausea and vomiting.

$>\mathrm{pHPT}$ is caused by a solitary adenoma in $85-90 \%$ of patients, and the curative treatment is parathyroidectomy.

$>$ Here, we presented two women who have diagnosed pHPT and operated without complications in pregnancy.

\section{CASE 1}

D She was 20 years old and presented with abdominal pain at 8th weeks gestation.

> Her laboratory tests were revealed $12.2 \mathrm{mg} / \mathrm{dl}$ Ca levels, 2 $\mathrm{mg} / \mathrm{dl}$ Phosphorous $(\mathrm{P})$ levels and $136 \mathrm{pg} / \mathrm{ml}$ parathyroid hormone (PTH) levels and her urine Ca level was 810 mg/24 hour.

$>$ Her neck ultrasonography (US) revealed a hypoechoic lesion with $6.3 \times 6.3 \times 14.5 \mathrm{~mm}$ size consistent with parathyroid adenoma in the left superior of the thyroid gland. No thyroid nodule was detected.

$>$ Nepfhrolithiasis was not determined

$>$ The left superior parathyroid gland was excised with minimal invasive surgery in the 9th weeks of gestation and parathyroid adenoma was excised.

\section{CASE 2}

$>38$ years old woman who was 9th weeks gestation was referred to our clinic for the high serum Ca levels.

> Her Ca level was 11.5 mg/dl, P level was $1.4 \mathrm{mg} / \mathrm{dl}$, PTH level was $344 \mathrm{pg} / \mathrm{ml}$.

$>$ Her neck US revealed parathyroid adenoma in the right inferior part of the thyroid gland $2 \times 1 \times 1 \mathrm{~cm}$ with size. Also she had a nodule in the right thyroid gland.

$>$ She had underwent right hemithyroidectomy and parathyroidectomy in the second trimester and parathyroid adenoma was excised.

\section{CONCLUSION}

D Since the symptoms are often nonspecific in the PHPT, it can be easily misdiagnosed during pregnancy.

$>$ Early recognition of $\mathrm{pHPT}$, followed by appropriate management and treatment may reduce the maternal and fetal complications

$>$ Therefore pregnant women with biochemical hypercalcemia or any clinical presentation associated with hypercalcemia must be evaluated for pHPT. 